\title{
A new, unique signature of the true cusp
}

\author{
W. R. Keith ${ }^{1}$, J. D. Winningham ${ }^{1}$, and O. Norberg ${ }^{2}$ \\ ${ }^{1}$ Southwest Research Institute, P. O. Drawer 28510, San Antonio, Texas 78228-0510, USA \\ ${ }^{2}$ Swedish Institute of Space Physics, Box 812, SE-981 28 Kiruna, Sweden
}

Received: 3 July 2000 - Revised: 13 April 2001 - Accepted: 18 April 2001

\begin{abstract}
The "cusp proper" is generally understood to be the region enclosed by the outermost magnetospheric field lines as they map to low altitudes. It is therefore a weak-field region with continuous contact with magnetosheath plasma. Data from the recent Astrid-2 Swedish microsatellite are presented which show a new, unique signature (dubbed the "true cusp") during cusp crossings that can now be shown to be consistently present and with which one can redefine the physical meaning and topology of the cusp. Similar crossings made by the DE-2, UARS and DMSP-F10 satellites also show this same, unique signature although in most cases the spatial resolution was much less than that of the Astrid-2 MEDUSA spectrometer. The presence of concurrent features of the same scale size as the plasma in the energetic particle, field and wave power data shows that this is a real structural feature and not a coincidental structure among plasma instruments. The persistence of this feature may lend new insight into the dynamics of the cusp and magnetospheric particle entry.
\end{abstract}

Key words. Magnetospheric physics (magnetopause, cusp, arid boundary layers; magnetospheric configuration and dynamics)

\section{Abbreviations}

DE, Dynamics Explorer;

DMSP, Defense Meteorlogical Satellite Program;

EMMA, Electric and Magnetic Monitoring of the Aurora;

HEPS, High Energy Plasma Spectrometer;

IGRF, International Geomagnetic Reference Field;

IL, Invariant Latitude;

IMF, Interplanetary Magnetic Field;

IRF, Swedish Institute for Space Physics;

IRFU, Swedish Institute for Space Physics - Uppsala

Division;

KTH, Swedish Royal Institute of Technology;

LAPI, Low Altitude Plasma Instrument;

Correspondence to: W. R. Keith (waynek@swri.edu)
LLBL, Low Latitude Boundary Layer;

MEDUSA, Miniaturized Electrostatic DUal-top-hat

Spherical Analyzer;

MEPS, Medium Energy Plasma Spectrometer;

MLT, Magnetic Local Time;

PEM, Particle Environment Monitor;

SwRI, Southwest Research Institute;

UARS, Upper Atmospheric Research Satellite.

\section{Introduction}

The 2-D and 3-D topology of the Earth's magnetosphere has long indicated the existence of a cusp, or weak magnetic field region, near magnetic local noon at the latitude where magnetic field lines switch from closing on the dayside to being swept back into the tail, allowing for more or less direct penetration of magnetosheath particle fluxes to low altitudes. Early observations (Heikkila and Winningham, 1971) showed a high-latitude band of low-energy particle precipitation with magnetosheath-like properties on the dayside at low-altitudes. They interpreted this feature as the longsought-for evidence of direct solar wind entry via a magnetic cusp. This general region of particle penetration was later separated into a "cusp proper" and a "Cleft/Boundary Layer", representing separate particle entry processes (i.e. direct and indirect) (Newell and Meng, 1988).

Newell and Meng (1988) defined the low-altitude cusp proper as the sub-region of plasma flux that more closely resembles magnetosheath plasma spectral characteristics, indicating "more direct" entry than that associated with the Low Latitude Boundary Layer (LLBL). This definition results in a cusp of much narrower extent in Magnetic Local Time (MLT) and Invariant Latitude (IL), and is limited to fairly direct plasma entry processes (i.e., little or no acceleration of the magnetosheath population). This smaller, more directly connected region is continuously present with a density that remains consistent with solar wind density variations (Aparicio et al., 1991). 
The true cusp can be defined in terms of the magnetopause current layer, which contains the outermost layer of magnetic field lines of the magnetosphere. This very thin layer is also the site of interconnection with interplanetary field lines and many other interesting processes, some of which are not well understood. The low-altitude regions to which these outermost field lines map are the magnetic cusps, which we believe to be associated with a small sub-region of the particledefined cusp mentioned above. Thus, this "true cusp" can represent a unique window into the large-scale workings of the magnetosphere and the magnetopause current layer. The primary difference between this definition and that of the cusp proper is that "true cusp" particles are expected to be more energetic, reflecting the accelerated populations seen in in situ measurements (Gosling et al., 1986; Song et al., 1990) and in simulations (Nakamura and Scholer, 2000) at the boundary.

Not long after the initial cusp observations, it was realized that the properties of the region were consistent with the theory of magnetic reconnection as proposed by Dungey (1968). The main property used to support this was the fact that the highest energy ions are seen at the most equatorward location, while lower energy particles tend to precipitate further poleward for southward IMF conditions (Shelley et al., 1976; Reiff et al., 1977). Magnetosheath plasma injected on the dayside at the reconnection point flows earthward along magnetic field lines. As the now open field line is convected tailward, it's footprint moves poleward. The higher energy particles reach low altitudes more quickly and, therefore, should be seen farther equatorward. This "dispersion" is the key to identifying reconnection, and has been reproduced by such models as the one of Lockwood and Smith (1994). So far, though, these models have not been able to reproduce all of the characteristics seen in the particle data (Fuselier et al., 1999).

The cusp proper averages approximately 2 to 3 hours wide in MLT and about 1 to $5^{\circ}$ in IL $\left(1^{\circ} \sim 100 \mathrm{~km}\right)$ (Newell and Meng, 1988; Lundin 1988; Aparicio et al., 1991) centered at noon and about $78^{\circ}$ IL. Its location and size vary with changes in the IMF direction and solar wind velocities, but it is always present (Newell and Meng, 1988). The above size and location represent statistical averages and are quite large. At low altitude orbits (1000 km polar circular), a feature of this size will be traversed on average in about $15 \mathrm{sec}-$ onds to a minute. Trajectories not cutting through the center of the cusp would have even shorter traversal times, on the order of a few seconds. Orbits with more equatorward inclinations may spend more time in the cusp, depending on how much local time is covered while inside the appropriate latitude range. A theoretical maximum for a cusp traversal (3 hours of local time at about $77^{\circ}$ latitude) would be on the order of 8 minutes. It is important to note that, while the quick traversals of the cusp at low altitude may have limited the detail seen in the past, they have the advantage of being more of a "snapshot" with less blurring of the lines between spatial and temporal features. The faster sample times of recent instruments (described below) allows the detail to be seen with- out the need to rely on higher altitudes and slower traversals, with the concomitant intermixing of populations due to horizontal transport. That is, mid-altitude measurements, while showing many similar particle characteristics, may contain bounced and tailward convected populations that may not accurately reflect the incoming particle population.

Historically, particle measurements of the cusp have been limited by the spatial resolution they could achieve, which is directly linked to the energy sweep rates of the instruments. Most detectors to date have had sweep rates on the order of a second, which for many low-altitude cusp crossings may allow for only a few energy spectrums to be taken if the structure is small. The latest instruments, however, including the MEDUSA instrument aboard Astrid-2, can take a 32-step energy sweep in 1/16 of a second for electrons and 1/8 of a second for ions, thus allowing unprecedented resolution $(\sim 400$ $\mathrm{m})$ of possible fine-scale structures.

\section{Instrument description}

The Miniaturized Electrostatic DUal-top-hat Spherical Analyzer (MEDUSA) was flown aboard the Swedish Astrid-2 microsatellite (Marklund, 2001). The instrument is composed of two spherical top-hat analyzers placed top-to-top with a common $360^{\circ}$ field of view. Each detector is divided into 16 azimuthal sectors of $22.5^{\circ}$, with an elevation acceptance of about $5^{\circ}$. It has an energy per charge range of about $1 \mathrm{eV}$ to $20 \mathrm{keV}$ for electrons and positive ions. The sensor and associated electronics box have a total mass of $1.7 \mathrm{~kg}$ and consume $3 \mathrm{~W}$ of power when in operation. More information on the MEDUSA instrument can be found in a paper by Norberg et al. (2001).

The MEDUSA analyzer can operate in two modes. In the first, data from all 16 sectors of both electrons and ions are relayed to the ground. In the second, however, only the three sectors from each side that are closest to the parallel, perpendicular and antiparallel magnetic field directions are recorded. The data presented below are of this second type. The spatial/temporal resolution is the same for these two modes and has been defined in the previous section. Given Astrid-2's circular orbit of $1000 \mathrm{~km}$, this gives a spatial resolution of $460 \mathrm{~m} / \mathrm{sweep}$ for electrons and $920 \mathrm{~m} / \mathrm{sweep} \mathrm{for}$ the ions.

Data are also presented from three other satellites which show similar cusp signatures. The first observation of this feature is from the LAPI instrument on the DE-2 satellite. From the UARS satellite, data are given for the HEPS/MEPS instruments. The US Air Force's SSJ/4 particle instrument on the DMSP-F10 satellite is the final source of data. In contrast to MEDUSA, these other instruments have sweep speeds ranging from 1 to 8 seconds.

\section{Observations}

The first example of this new cusp feature comes from a cusp pass of the DE-2 satellite on 6 September 1982 (Fig. 1). 


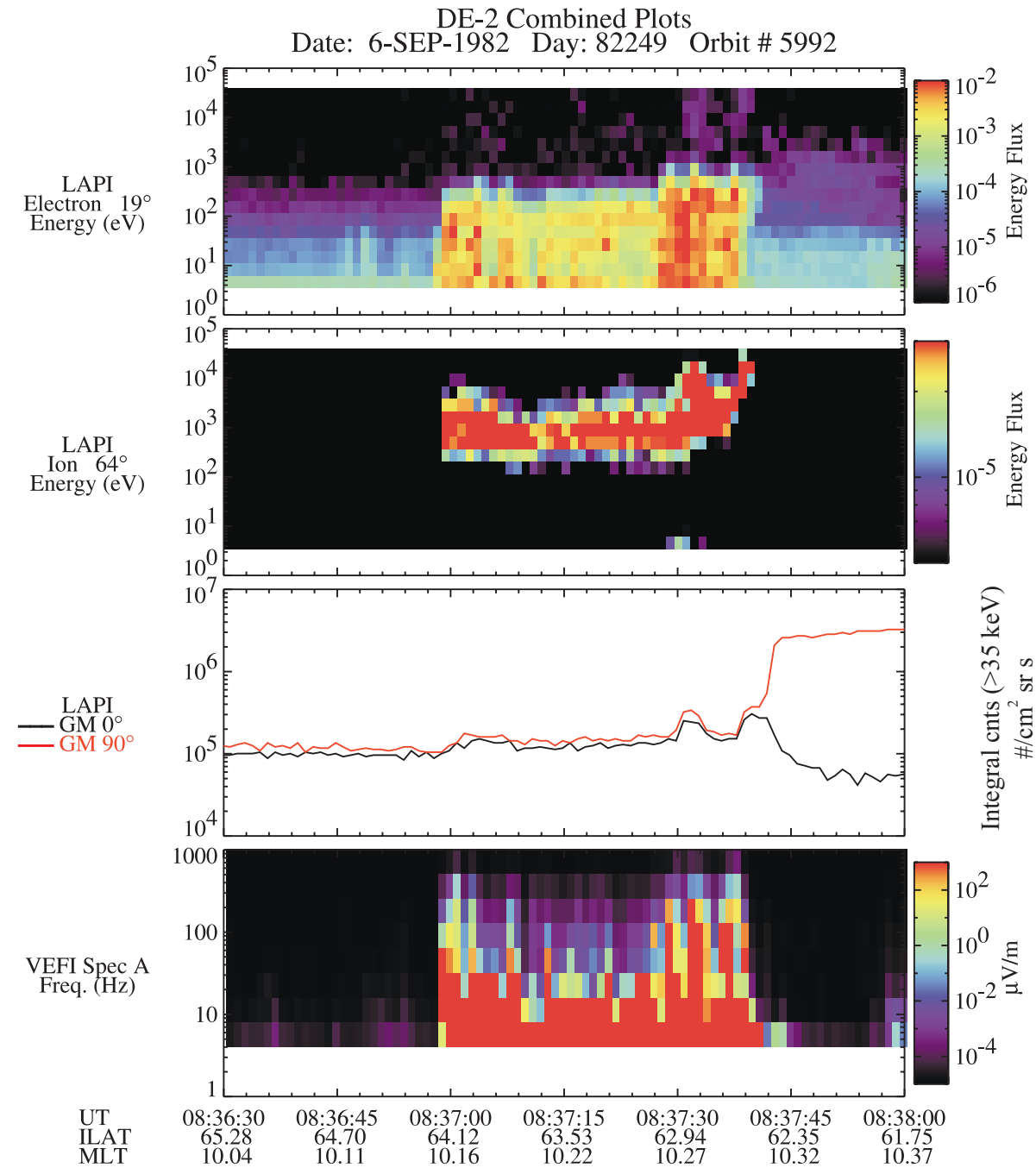

Fig. 1. DE-2 data from 6 September 1982 showing a cusp dispersion signature in the ions with a small $\mathrm{V}$ at the equatorward end. Top panel is electron energy flux, second is ion energy flux. The line plots in the third panel are counts of $>35 \mathrm{keV}$ electrons in the parallel (black) and perpendicular (red) directions. The bottom spectrogram is electric field wave power up to $1 \mathrm{kHz}$.
The data were taken by the Low Altitude Plasma Instrument (LAPI); this consists of 15 parabolic electrostatic analyzers covering $180^{\circ}$ for ions and electrons from $5 \mathrm{eV}$ to $32 \mathrm{keV}$ and two Geiger-Mueller counters which measure $>35 \mathrm{keV}$ electrons at $0^{\circ}$ and $90^{\circ}$ pitch angles. One 32-step spectrum is taken each second from each sensor. Detailed information on this instrument is available from Winningham et al. (1981). The data were taken on an equatorward pass, the cusp being located at an Invariant Latitude of $63^{\circ}$ and an MLT pre-noon (10:13). A "typical looking" dispersion signature can be seen in the ion data (second panel), moving from lower (poleward) energies to higher (equatorward) energies, although the spectrogram appears to be flat to first order at about $1 \mathrm{keV}$ from $8: 37: 00$ to $8: 37: 30$ (all times given in UT). The feature we are interested in, however, is less than a third of the "typical" dispersion pattern, at the equatorward edge from 8:37:30 to 8:37:40. This 10 -second interval clearly has a $\mathrm{V}$ shaped ion structure with higher energies on the edges and lower energies towards the center. This $\mathrm{V}$ is at unusually high energies, with peaks at around $20 \mathrm{keV}$ down to $2 \mathrm{keV}$ at the center. The electrons (Fig. 1, top panel) clearly show enhancement during this same time period, from 30 kilovolts down to the lowest energies. Above $1 \mathrm{keV}$, the fluxes vary spatially the same as the GM data in panel 3. Below $1 \mathrm{keV}$ there is a different morphology. The third panel of Fig. 1 is GeigerMueller data and the parallel (black) and perpendicular (red) lines both indicate clear enhancements over their already elevated levels at the two edges of the feature and are collocated with the ion V edges. The bottom panel of Fig. 1 is AC electric wave power from the VEFI instrument displayed from 4 to $1000 \mathrm{~Hz}$. This spectrogram shows strong waves at 400 $\mathrm{Hz}$ and lower during the $\mathrm{V}$. The ion feature covers about $0.4^{\circ}$ in IL and about 18 minutes of MLT, which corresponds to a very narrow feature of approximately $50 \mathrm{~km}$.

Data from the MEDUSA and EMMA instruments aboard Astrid-2 are shown in Fig. 2. The data were taken on 13 January 1999, during a pass over the southern auroral zone. The spacecraft was traveling equatorward at an altitude of $1029 \mathrm{~km}$. In the antiparallel (downward moving) ion spectrogram (Fig. 2, bottom panel), another typical dispersion signature can be seen, although it appears to be nearly flat as well at about $300 \mathrm{eV}$ from 20:12:49 to 20:13:13. The V 

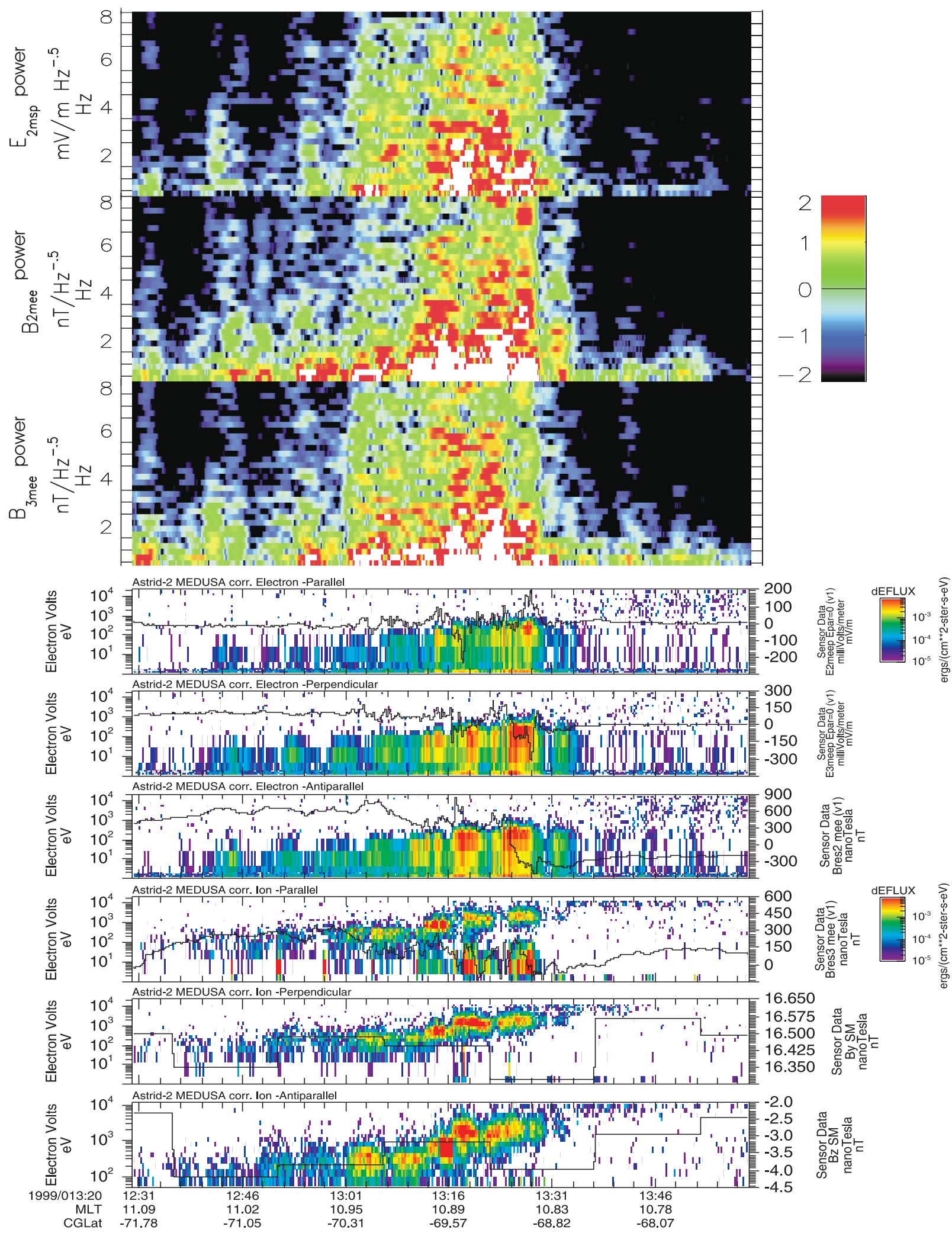

Fig. 2. Astrid-2 data from 13 January 1999 with a dispersion signature and equatorward V similar to DE-2. Top spectrogram is electric field wave power up to $8 \mathrm{~Hz}$. The second and third panels are delta magnetic field wave power. The middle three spectrograms are electrons in the parallel, perpendicular, and antiparallel directions, respectively. The bottom three spectrograms are for the same three look directions for the ions. The line plots in the fourth and fifth panels are electric field calculated for the eastward and equatorward directions, assuming $E$ is zero along the magnetic field. The two panels below it contain delta magnetic field data for the same two directions. The bottom two panels contain the IMF in the $y$ and $z$ directions as measured by IMP-8. 
feature is less than a third of this "typical" dispersion pattern, at the equatorward edge from 20:13:16.5 to 20:13:28. The onset of the feature stands out as a sudden increase of about $1.5 \mathrm{keV}$ (from $\sim 500 \mathrm{eV}$ to $\sim 2 \mathrm{keV}$ ) in the peak energy of the ions. The peak energy of the feature then dips to about $1 \mathrm{keV}$ at 20:13:21.5 and becomes weaker in intensity. From this point until 20:13:28 the energy steadily increases back to $2 \mathrm{keV}$. The ions cut off abruptly on the equatorward edge of the cusp at 20:13:30. The same feature is present in the mirroring perpendicular pitch-angle ions, and the $2 \mathrm{keV}$ "wings" appear in the parallel (upwards) ions, however, the lower energy central portion is not present.. In these latter two spectrograms (second and third from the bottom), lowenergy enhancements $(\sim 3 \mathrm{eV}$ to $100 \mathrm{eV})$ can also be seen at the beginning $(20: 13: 18)$ and end $(20: 13: 25)$ of the feature moving up the field line. The antiparallel (downwards) electrons (and to a lesser extent the other pitch-angle electrons) also show $<300 \mathrm{eV}$ enhancements at 20:13:18 and 20:13:25 (middle three panels).

The EMMA electric and magnetic field experiment measures two spin-plane components of the electric field and three dimensions of magnetic field. For the data presented here, the electric field along the direction of the magnetic field has been assumed to be zero and the two perpendicular components in the eastward and equatorward directions have been calculated. The magnetic field direction makes an angle of $-23^{\circ}$ with the spacecraft spin-plane at the time of this cusp crossing. The electric field in the fourth (eastward) panel fluctuates about $-10 \mathrm{mV} / \mathrm{m}$ outside of the cusp but begins to gain amplitude going into the low-energy end of the dispersion feature at about 20:13:00. The field peaks to -225 $\mathrm{mV} / \mathrm{m}$ at the poleward edge of the ion feature at 20:13:16.5. After this, it increases steadily to $200 \mathrm{mV} / \mathrm{m}$ at the equatorward end of the feature (20:13:28), and afterwards settles to 0 . The equatorward (north, in this case) pointing perpendicular electric field in the fifth panel begins at a potential of $100 \mathrm{mV} / \mathrm{m}$ before dropping by $250 \mathrm{mV} / \mathrm{m}$ at the poleward edge of the V. It then returns to its original value of 100 $\mathrm{mV} / \mathrm{m}$ in the center before plunging to $-300 \mathrm{mV} / \mathrm{m}$ at the far edge of the feature. It then settles to approximately zero as well. The delta magnetic field, calculated by subtracting the standard IGRF 95 geomagnetic model field from the full field measurements, is shown on the fourth and third from the bottom panels of Fig. 2 in the same two directions as were displayed for the electric field. The upper (eastward) component goes from $600 \mathrm{nT}$ before the $\mathrm{V}$, down to -150 $\mathrm{nT}$ at the end, revealing a significant current system in that one limited region. Northward data in the lower panel also shows a net drop of about $200 \mathrm{nT}$ over the feature, although the trend is much less obvious in this case. The bottom two panels contain the GSM y and z components of the Interplanetary Magnetic Field, as measured by IMP-8 outside of the bowshock. These values have not been time-shifted; however they are representative of the delayed values for this pass (weakly southward and strongly duskward). The possible significance of this will be explored in the discussion section. The magnetic wave power (Fig. 2, second and third panels) is calculated by a FFT from the detrended magnetometer data (lower two panels) perpendicular to the main field in the eastward (second spectrogram) and equatorward (third spectrogram) directions. The electric field wave power is calculated in the same manner and is shown at the top. The spectrograms show frequencies up to $8 \mathrm{~Hz}$, calculated with a four second sliding window. The time period of the ion feature, from 20:13:16 to 20:13:28, coincides with a dramatic increase of over two orders of magnitude in the wave power at all displayed frequencies.

The Magnetic Local Time of the ion V event was about $10: 52$, and the Invariant Latitude range covered only $0.57^{\circ}$, from $-69.54^{\circ}$ to $-68.97^{\circ}$. The north/south component of the satellite track as it crossed this feature is only $\sim 74 \mathrm{~km}$ (only about 10 of the old one-second sweeps) which makes this a very small feature indeed, even in terms of the cusp proper. Examples of this type of feature are by no means unique, but can be hard to spot due to their small size and the fact that they are very localized in IL and MLT, being found within about an hour of noon in MLT at cusp latitudes.

Other instruments have seen similar structures, despite the fact that they typically have much lower time resolutions. The HEPS instrument on the UARS satellite studies highenergy electrons and ions (from $30 \mathrm{keV}$ to $5 \mathrm{MeV}$ and 150 $\mathrm{MeV}$, respectively) and consists of four electron/proton detectors, two electron detectors and two Low Energy Proton (LEP) detectors (Winningham et al., 1993). HEPS data presented here (Fig. 3) are electrons from one of the near-zenith pointing detectors and from one of the LEP detectors. The MEPS spectrometer is made up of eight divergent plate electrostatic analyzers and looks at electrons and ions in the $1 \mathrm{eV}$ to $32 \mathrm{keV}$ range. Each detector is situated on the spacecraft to have a different look direction angle with respect to the spacecraft. The MEPS data here are electrons and ions from the detector with a look direction of $36.6^{\circ}$ with respect to the spacecraft zenith (Winningham et al., 1993). Data from a 1991 cusp pass are presented in Fig. 3. The data were taken on 9 November, while UARS was heading poleward at an altitude of about $600 \mathrm{~km}$. The feature of interest is located in the afternoon (13:30) sector in MLT and about $67^{\circ}$ IL (about $2^{\circ}$ lower in IL than the previous Astrid-2 data). The time resolution is 2 seconds for MEPS and 4 and 8 for HEPS electrons and protons, respectively. A V shaped feature can be distinguished which lasts about 40 seconds in the MEPS ion data (second panel); this begins at 05:40:40 at about $2 \mathrm{keV}$, decreases to $\sim 500 \mathrm{eV}$ in the center $(05: 40: 56)$ and increases back to $2 \mathrm{keV}$ at 05:41:17. The MEPS electron data over this same time period shows enhancements centered at about $40 \mathrm{eV}$ at the beginning and end, with a strong flux of $100 \mathrm{eV}$ electrons throughout the event. Notice the softening of the electrons at the ends, where the density is the highest. HEPS high-energy particle data also shows significant enhancements at the edges of the feature at all energies up to the max plotted of about $300 \mathrm{keV}$. Equatorward of the feature, both electrons and ions show very large fluxes. From 05:40:26 to 05:40:43, the HEPS electrons fall off steadily, corresponding to the beginning of the MEPS ion 


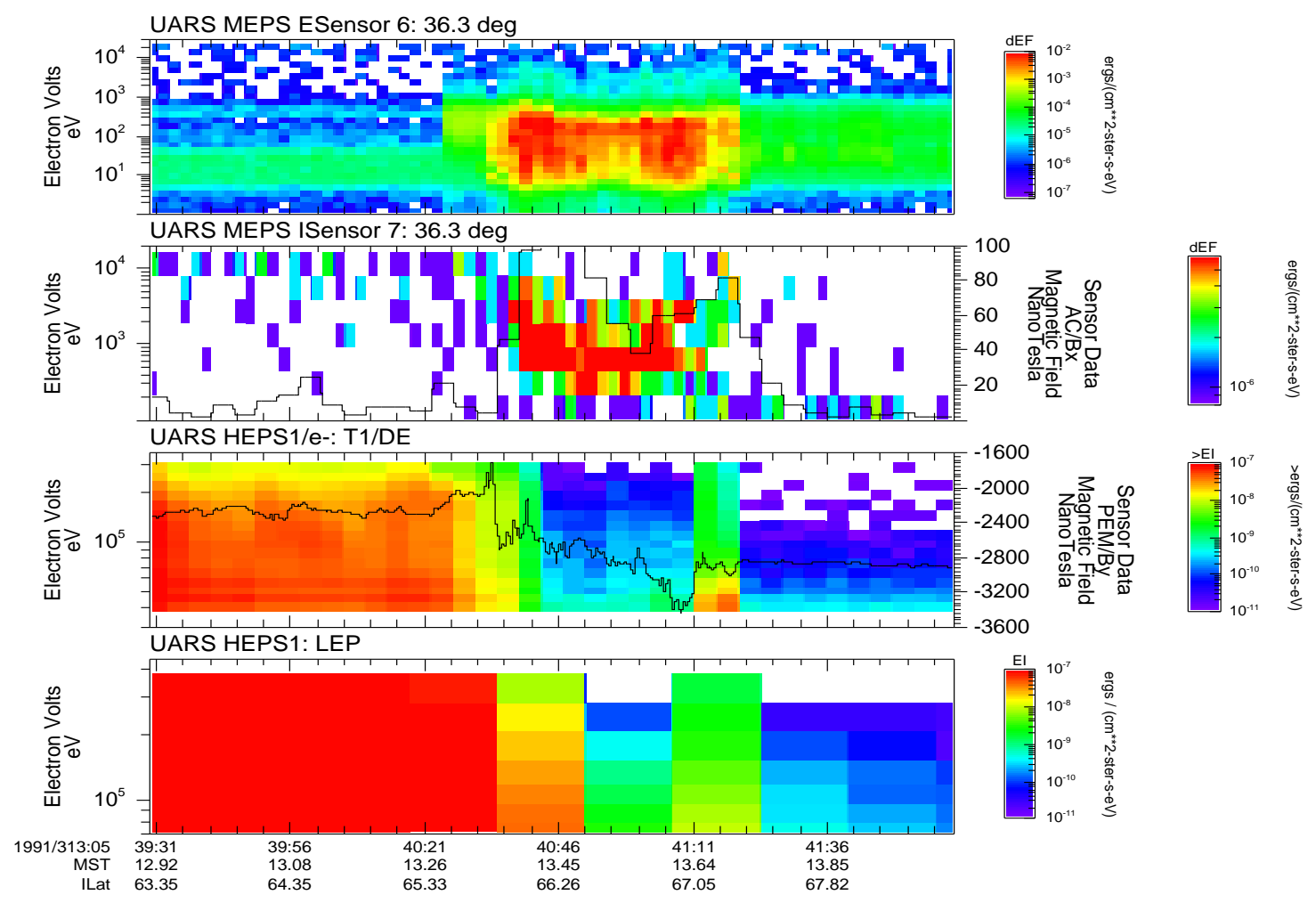

Fig. 3. UARS data from 9 November 1991, showing a V signature without the dispersion tail. Top panel is electrons $36^{\circ}$ from zenith and the second panel is ions from the same direction. The third spectrogram is electrons from $35 \mathrm{keV}$ up to $300 \mathrm{keV}$. The bottom panel contains ions from $70 \mathrm{keV}$ to $400 \mathrm{keV}$. The line plot in the second panel is integrated magnetic field wave power from 0 to $100 \mathrm{~Hz}$ and the third panel has a line plot of the $B_{y}$ magnetic field.

feature. There is also a significant increase of electrons at the poleward edge of the feature at 05:41:16, which peaks at $40 \mathrm{keV}$. The LEP protons decrease at the onset of the feature similarly to the HEPS electrons and also show an increase at all energies at the opposite end (05:41:16).

The $B_{y}$ magnetic field (line plot in third from top panel), which is fairly constant before and after the feature, is very disturbed during the period from 05:40:40 to 05:41:17, increasing sharply and then decreasing steadily during this time period. The magnetic wave power data is integrated from 0 to $100 \mathrm{~Hz}$ and labeled $\mathrm{AC} / \mathrm{Bx}$ (on the right of the second panel). The integrated power increases by over an order of magnitude to $100 \mathrm{nT}$ at the onset of the ion feature, dips down to about 40 near the center at 05:41:01 and then peaks again at $80 \mathrm{nT}$ at the end of the structure before returning to low (under $10 \mathrm{nT}$ ) values. The Invariant Latitude covered during this time, however, is only $1.2^{\circ}$ which is about $143 \mathrm{~km}$, a factor of 2 larger than the Astrid- 2 feature but still at the very bottom of the range of cusp sizes.

Particle data from the SSJ/4 instrument of the DMSP-F10 satellite can be seen in Fig. 4. SSJ/4 is a set of four cylindrical electrostatic analyzers, two sensors (high and low energies) for electrons and ions. Together they cover an energy range from $30 \mathrm{eV}$ to $30 \mathrm{keV}$, completing a spectrum once per second. Further information about the DMSP program and the SSJ/4 instruments can be found in Hardy et al. (1984).
The data presented are from 28 March 1992, when the satellite was passing equatorward over the cusp at an Invariant Latitude of $73^{\circ}$ and a MLT just post noon (12:20). The ion data again exhibits the energy-latitude dispersion as it passes from higher to lower latitudes and also clearly shows a "V" structure, beginning at 10:10:55 UT, which lasted just under 25 seconds. The peak energy of the poleward edge of the structure is slightly less in this case, about $1 \mathrm{keV}$, however the middle and equatorward edges are peaked at $500 \mathrm{eV}$ and $2 \mathrm{keV}$ as in the previous two cases. The corresponding electron enhancements at the edges are also similar to the previous cases, being mostly in the 100 to $200 \mathrm{eV}$ range. The satellite altitude at the time of the pass was $770 \mathrm{~km}$ and the feature spanned $0.77^{\circ}$ of invariant latitude, giving it a length of about $96 \mathrm{~km}$ which, like the other cases, is very small; on the order of a proton gryroradius at the magnetopause.

\section{Discussion}

We have presented data from several different satellites and instruments, representing various sensor types and technologies. The fact that the narrow "V" feature is consistent throughout all of the data sets from different satellites and plasma analyzer types indicates that it is not a characteristic of the instruments taking the data, and must be consid- 

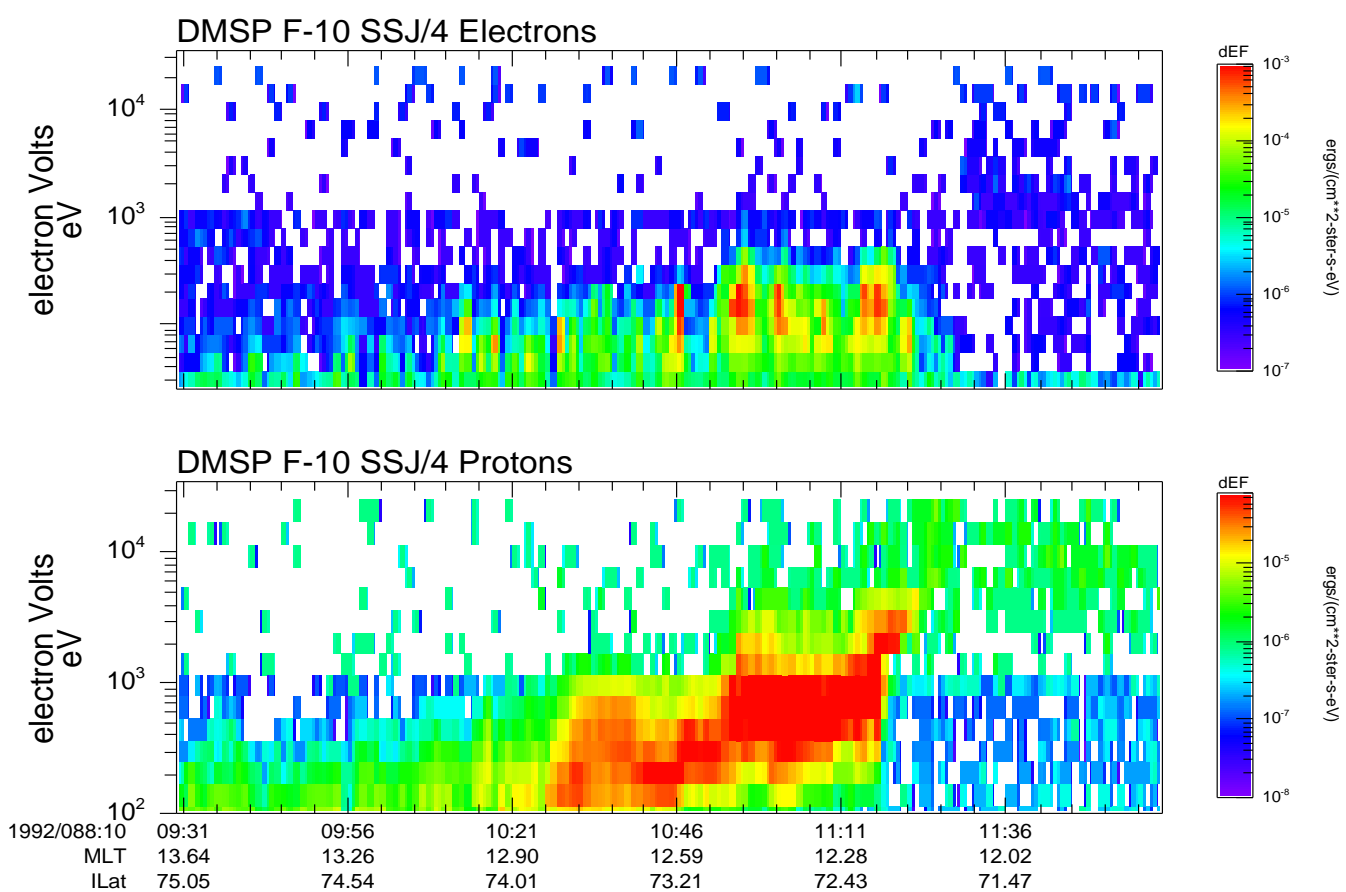

Fig. 4. DMSP F-10 data from 28 March 1992; a dispersion signature can be seen along with a lopsided V. The upper spectrogram is electrons, while the lower is ions.

ered to be a real structure. Also, the fact that the energetic particle and field instruments see a feature at the same time with a comparable scale-size also points to this being something physical. The feature is also persistent; the examples that have so far been found with this distinctive shape have prompted a more thorough statistical search of Astrid-2 and DMSP data. The search will be conducted among passes whose orbital track passes through a box containing Magnetic Local Times between 10:30 and 13:30 and Invariant Latitudes between $60^{\circ}$ and $80^{\circ}$ or $-60^{\circ}$ and $-80^{\circ}$.

Reiff et al. (1977) discuss an injection model, called "diffusive injection", which assumes an energy dependence on the rate of diffusion from a central "injection field line". This results in an energy dispersion in which the highest energy particles are towards the edges of the cusp (since they can random-walk farther from their injection point than the lower energy particles). Later works (Reiff et al., 1980; Burch et al., 1980; Woch and Lundin 1992; Weiss et al., 1995) confirmed that these features are seen, usually during northward IMF conditions, and offered other explanations for these "large scale V's". Possible explanations, such as tail reconnection, changes in the IMF $B_{z}$ and lobe reconnection have been offered. The features presented here, however, are of a much smaller scale size than the large $\mathrm{V}$ dispersion features studied by these authors and appear to be very localized to what has been defined above to be the "true cusp" region. While some characteristics and possible causes of these two structures may overlap, we believe that they are not equivalent.

If this feature is the "true cusp" as defined above, then it is simply the low-altitude mapping of the magnetopause cur- rent layer. The current layer is the boundary that separates the magnetosphere which is dominated by the Earth's geomagnetic field and the magnetosheath which is dominated by the IMF from the Sun. Higher energy particles penetrate more deeply into this boundary layer and so will be on the earthward side of the current layer while lower energy particles are closer to the magnetosheath. Song et al. (1990) reported that the ion and electron distribution functions increased in average energy, moving inwards at the current layer. At low altitudes, the "inner" edge of the magnetopause is towards the edges of the true cusp while the lower energy outer parts map towards the center (Stasiewicz, 1991). The result is a low-altitude energy signature that is peaked at the edges of the cusp, or "V" shaped, reflective of ion energization in the current layer that is known to increase from outer to inner edge. The closer to the center of the cusp the satellite track is, the deeper and wider the V. The collocation of the energetic particles and increase in wave power are also clues to the origin of the feature, although a simple "cartoon" picture of the magnetopause quickly becomes insufficient to explain them. The observed low-frequency waves may be those that are seen in the magnetopause current layer (Gurnett et al., 1979), traveling down the field lines as a wave guide and further supporting the idea that this is a low-altitude mapping of the turbulent current layer. The high-energy particles seen at the poleward edge of the UARS event are more puzzling. If the field lines in this region do not remain high in the plasma mantle, as in the classical picture, but instead twist to lower latitudes in the tail (as can sometimes be seen in the Tsyganenko96 model) these high energy particles may be part of the drifting population that is lost on the flanks of the magne- 


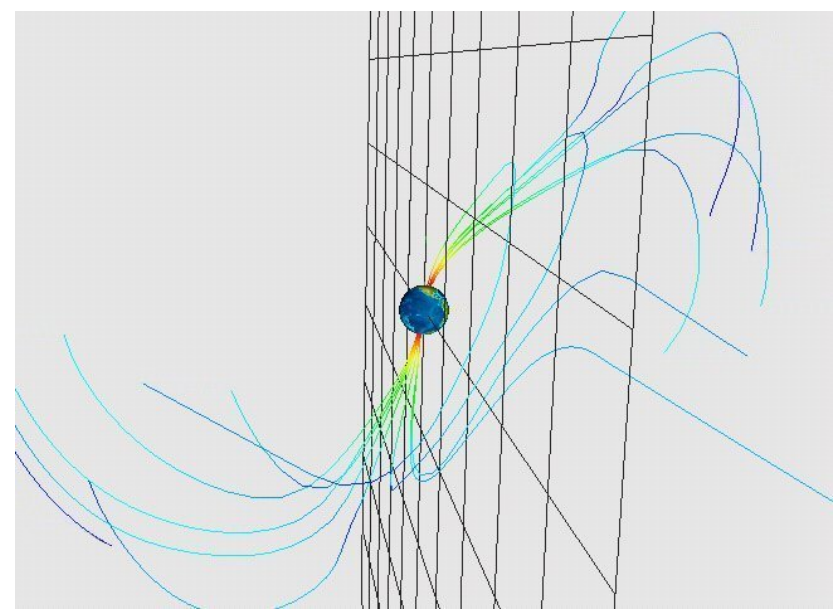

Fig. 5. Screen dump from the Orbit Visualization Tool showing the Earth, the outermost set of magnetic field lines as calculated from the Tsyganenko96 model and the field line passing though Astrid-2 (bottom side of the Earth, not shown). Note the twisting of the field lines relative to the $x-z$ plane shown.

topause. If this magnetopause mapping is indeed the source of the signature, then this opens up a new technique in the study of magnetopause dynamics.

A preliminary study of the January 1999, Astrid-2 pass has been conducted using a pre-release version of Orbit Visualization Tool (OVT) being developed at the Swedish Institute of Space Physics in Uppsala (IRFU) by a team lead by Kristof Stasiewicz. This software includes 3-D visualization of T96 generated magnetospheric field lines (Tsyganenko, 1995) and satellite orbits. Magnetopause field lines (adjusted for IMF and plasma pressure) during this cusp pass in January 1999, are in good agreement with the satellite position (Fig. 5) and cover a low-altitude area in IL and MLT that is consistent with the observations. The field line passing through the satellite location is the one that exits the figure on the lower right hand side. This time step is near the beginning of the pass. Subsequent time steps are closed and move inwards with the progression of time. This visualization, of course, cannot unambiguously determine the open/closed field line boundary during the pass; however, it serves to illustrate the relative positions of the field lines crossed. Note also the dawn/dusk warping of the cusp field lines relative to the $(x-z$ plane $)$ grid. One interesting characteristic of the field lines during the Astrid-2 pass is their obvious twisting in the GSM $y$ direction. This is due to the fact that the IMF input into the Tsyganenko model for that time was strongly dominated by $B_{y}$ (see Fig. 2, bottom two panels). Further investigation revealed that all of the V's (for which IMF data is available) took place when the IMF had a dominant $B_{y}$ component. This possible IMF dependence will need to be explored in greater detail but offers a further explanation for the double-sided nature of the V's. Crooker (1988) models the cusp as a wedge whose base is fixed while the tip rotates with changing IMF $B_{y}$. The edges of the wedge map to the newest open field lines, or $x$-line, at the outer surface of the magnetopause. During strong $B_{y}$, which has been the case for all of the V's studied so far, the cusp will be "wind socked" over so that the spacecraft in a polar orbit would cross it sideways giving a double signature, the second part being the mirror of the first. This is reflective of the two disconnected $X$-lines on the dawn/dusk sides in Crooker's model. Available electric field data in the current set of observations appears to support this interpretation. The electric field is V-shaped about the centerline and does not change direction. The integrated potential for the Astrid-2 pass is about $50 \mathrm{kV}$; this does not represent the maximum, as we do not cross the maximum contours. This is consistent with Crooker's idea of a concentrated electric field in the throat.

\section{Conclusions - future work}

This paper presents a previously unreported small-scale feature that appears to be unique to regions we call the true cusps of the northern and southern hemispheres. It is hoped that as more and more high-resolution low-altitude data, such as that from the MEDUSA instrument, becomes available, new information about the cusp and the associated particle entry processes may be derived. The November 2000 launch of Munin, whose primary payload is MEDUSA-2, should add greatly to the dataset of MEDUSA-class instruments and yield many new cusp passes for study.

It is clear that more work needs to be done in studying these features and the connections between the true cusp, the magnetopause current layer and the Interplanetary Magnetic Field in general. Future work, which will comprise a $\mathrm{PhD}$ dissertation, will focus on understanding the connections between the IMF, magnetopause current layer and the cusp as well as increasing the statistical significance of the feature by searching for more occurrences. In addition, the OVT 3dimensional visualization tool being developed will be used to characterize the field line geometry at the satellite location at the time the data was taken. It is hoped that this work may lead to a consensus on particle entry methods and the relationship of the magnetopause current layer to the true cusp.

In summary, the data presented are consistent with a measurable low altitude image of the magnetopause current layer. This set of field lines are those on which ions are accelerated above magnetosheath energies and which form the classical magnetic cusp (i.e. not a plasma cusp). The mapping topology at low altitudes appears to "swing" with $B_{y}$ as expected from the Crooker model and has a concentrated electric field as predicted. The wave data are also consistent with the observations of a turbulent, noisy magnetopause in the ELF/ULF region. Energetic particles could be dumped drifting particles which are on field lines that see a non-equatorial mirror force (Sheldon et al., 1998; Delcourt and Sauvaud, 1999) in the vicinity of the magnetopause and execute drift up and into the magnetic cusp where they circulate and can be lost. 
Acknowledgements. The authors would like to acknowledge our MEDUSA and Astrid-2 partners in Sweden, without whom this instrument would not have flown; especially the MEDUSA team at the Swedish Institute for Space Physics (IRF) in Kiruna and the Astrid-2/EMMA team at the Royal Institute of Technology (KTH) in Stockholm. This work was supported by ONR contract N0001498-1-0175.

Topical Editor G. Chanteur thanks K. Stasiewicz and another referee for their help in evaluating this paper.

\section{References}

Aparicio, B., Thelin, B., and Lundin, R., The polar cusp from a particle point of view: A statistical study based on Viking data, J. Geophys. Res., 96, 14023, 1991.

Burch, J. L., Reiff, P. H., Heelis, R. A., Spiro, R. W., and Fields, $\mathrm{S}$. A., Cusp region particle precipitation and ion convection for northward interplanetary magnetic field, Geophys. Res. Lett., 7, 393,1980

Crooker, N. U., Mapping the merging potential from the magnetopause to the ionosphere through the dayside cusp, J. Geophys. Res., 93, 7338, 1988.

Delcourt, D. C. and Sauvaud, J.-A., Populating of the cusp and boundary layers by energetic (hundreds of $\mathrm{keV}$ ) equatorial particles, J. Geophys. Res., 104, 22635, 1999.

Dungey, J. W., Waves and particles in the magnetosphere, in The Physics of the Magnetosphere, Eds. R. L. Carovillano, J. F. McClay and H. R. Radoski, p. 246, D. Riedel, Norwell, Mass., 1968.

Fuselier, S. A., Lockwood, M., Onsager, T. G., and Peterson, W. K., The source population for the Cusp and Cleft/LLBL for southward IMF, Geophys, Res. Lett., 26, 1665, 1999.

Gosling, J. T., Thomsen, M. F., Bame, S. J., and Russell, C. T., Accelerated plasma flows at the near-tail magnetopause, J. Geophys. Res., 91, 3029, 1986.

Gurnett, D. A., Anderson, R. R., Tsurutani, B. T., Smith, E. J., Paschmann, G., Haerendel, G., Bame, S. J., and Russell, C. T., Plasma wave turbulence at the magnetopause: Observations from ISEE 1 and 2, J. Geophys. Res., 84, 7043, 1979.

Hardy, D. A., Schmitt, L. K., Gussenhoven, M. S., Marshall, F. J., Yeh, H. C., Schumaker, T. L., Huber, A., and Pantazis, J., Precipitating electron and ion detectors (SSJ/4) for the block 5D/flight 6-10 DMSP satellites: Calibration and data presentation, Rep. AFGL-TR-84-0314, Air Force Geophys. Lab., Hanscom Air Force Base, Mass., 1984.

Heikkila, W. J. and Winningham, J. D., Penetration of magnetosheath plasma to low altitudes through the dayside magnetospheric cusps, J. Geophys. Res., 76, 883, 1971.

Lockwood, M. and Smith, M. F., Low and middle altitude cusp particle signatures for general magnetopause reconnection rate variations: 1. Theory, J. Geophys. Res., 99, 8531, 1994.

Lundin, R., On the magnetospheric boundary layer and solar wind energy transfer into the magnetosphere, Space Sci. Rev., 48, 263, 1988.

Marklund, G. T., Blomberg, L. G., and Persson, S., Astrid-2, an advanced microsatellite for auroral research, Ann. Geophysicae, 19, 589-592, 2001 (this issue).

Nakamura, M. and Scholer, M., Structure of the magnetopause reconnection layer and of flux transfer events: Ion kinetic effects, J. Geophys. Res., 105, 23179, 2000.

Newell, P. T., and Meng, C.-I., The cusp and cleft/boundary layer: Low altitude identification and statistical local time variation, J. Geophys. Res., 93, 14549, 1988.

Norberg, O., Winningham, J. D., Lauche, H., Keith, W., Puccio, W., Olsen, J., Lundin, K., and Scherrer, J., The MEDUSA electron and ion spectrometer and the PIA photometers on Astrid-2, Ann. Geophysicae, 19, 593-600, 2001 (this issue).

Reiff, P. H., Hill, T. W., and Burch, J. L., Solar wind plasma injection at the dayside magnetospheric cusp, J. Geophys. Res., 82, 479, 1977.

Reiff, P. H., Burch, J. L., and Spiro, R. W., Cusp proton signatures and the interplanetary magnetic field, J. Geophys. Res., 85, 5997, 1980.

Sheldon, R. B., Spence, H. E., Sullivan, J. D., Fritz, T. A., and Chen, J., The discovery of trapped energetic electrons in the outer cusp, Geophys. Res. Lett., 25, 1825, 1998.

Shelley, E. G., Sharp, R. D., and Johnson, R. G., $\mathrm{He}^{++}$and $\mathrm{H}^{+}$ flux measurements in the dayside cusp: Estimates of convection electric field, J. Geophys. Res., 81, 2363, 1976.

Song, P., Elphic, R. C., Russell, C. T., Gosling, J. T., and Cattell, C. A., Structure and properties of the subsolar magnetopause for northward IMF: ISEE observations, J. Geophys. Res., 95, 6375, 1990

Stasiewicz, K., Polar cusp topology and position as a function of interplanetary magnetic field and magnetic activity: Comparison of a model with viking and other observations, J. Geophys. Res., $96,15789,1991$

Tsyganenko, N. A., Modeling the Earth's magnetospheric magnetic field confined within a realistic magnetopause, J. Geophys. Res., 100, 5599, 1995

Weiss, L. A., Reiff, P. H., Webber, E. J., Carlson, H. C., Lockwood, M., and Peterson, W. K., Flow-aligned jest in the magnetospheric cusp: Results from the Geospace Environment Modeling Pilot program, J. Geophys. Res., 100, 7649, 1995.

Winningham, J. D., Burch, J. L., Eaker, N., Blevins, V. A., and Hoffman, R. A., The Low Altitude Plasma Instrument (LAPI), Space Sci. Inst., 5, 465, 1981.

Winningham, J. D., Sharber, J. R., Frahm, R. A., Burch, J. L., Eaker, N., Black, R. K., Blevins, V. A., Andrews, J. P., Rudski, J., Sablik, M. J., Chenette, L. D., Datlowe, D. W., Gaines, E. E., Imhof, W. I., Nightingale, R. W., Reagan, J. B., Robinson, R. M., Schumaker, T. L., Shelley, E. G., Vondrak, R. R., Voss, H. D., Bythrow, P. F., Anderson, B. J., Potemra, T. A., Zanetti, L. J., Holland, D. B., Rees, M. H., Lummerzheim, D., Reid, G. C., Roble, R. G., Clauer, C. R., and Banks, P. M., The UARS Particle Environment Monitor, J. Geophys. Res., 98, 10649, 1993.

Woch, J. and Lundin, R., Magnetosheath plasma precipitation in the polar cusp and its control by the interplanetary magnetic field, J. Geophys. Res., 97, 1421, 1992. 